INPLASY

PROTOCOL

To cite: Li et al. Benefits and risks of metformin use in patients with COVID-19: A protocol for systematic review and meta-analysis. Inplasy protocol 202120055. doi: 10.37766/inplasy2021.2.0055

Received: 18 February 2021

Published: 18 February 2021

Corresponding author:

Zhi Zeng

zhizeng_scu@yeah.net

Author Affiliation:

West China hospital, Sichuan

University, China

Support: None.

Review Stage at time of this submission: The review has not yet started.

Conflicts of interest:

None declared.

\section{Benefits and risks of metformin use in patients with COVID-19: A protocol for systematic review and meta- analysis}

\author{
Li, Y1; Sun, T2; Yang, X3; Yan, P4; Zeng, Z5.
}

Review question / Objective: The objective of this work is to test the benefits and harms of the use of metformin in patients with COVID-19.

Condition being studied: The novel coronavirus disease 2019 (COVID-19), caused by severe acute respiratory syndrome coronavirus 2 (SARS-CoV-2), has become a critical public health issue throughout the world. Early evidence has suggested age, gender, obesity, and comorbidities are likely associated with COVID-19 outcomes. More importantly, subsequent studies identified diabetes mellitus as an independent risk factors for mortality, severity, and morbidity of patients with COVID-19. Thus, the benefits of glucoselowering medication in patients with COVID-19 have been repeatedly tested. As one of the most widely used antidiabetic drugs in patients with diabetes, metformin appeared to favorably influence the COVID-19 outcomes, particularly attenuating the risk of death. However, the potential benefits and risk of metformin use in patients with COVID-19 are not clear.

INPLASY registration number: This protocol was registered with the International Platform of Registered Systematic Review and Meta-Analysis Protocols (INPLASY) on 18 February 2021 and was last updated on 18 February 2021 (registration number INPLASY202120055).

\section{INTRODUCTION}

Review question / Objective: The objective of this work is to test the benefits and harms of the use of metformin in patients with COVID-19.
Condition being studied: The novel coronavirus disease 2019 (COVID-19), caused by severe acute respiratory syndrome coronavirus 2 (SARS-CoV-2), has become a critical public health issue throughout the world. Early evidence has suggested age, gender, obesity, and 
comorbidities are likely associated with COVID-19 outcomes. More importantly, subsequent studies identified diabetes mellitus as an independent risk factors for mortality, severity, and morbidity of patients with COVID-19. Thus, the benefits of glucose-lowering medication in patients with COVID-19 have been repeatedly tested. As one of the most widely used anti-diabetic drugs in patients with diabetes, metformin appeared to favorably influence the COVID-19 outcomes, particularly attenuating the risk of death. However, the potential benefits and risk of metformin use in patients with COVID-19 are not clear.

\section{METHODS}

Search strategy: We will search literatures in the following databases: PubMed, Embase, Cochrane Library, China National Knowledge Infrastructure (CNKI), Wan fang, and Chinese Biomedical Literature Database (CBM), from its establishment to Dec 31, 2020. We will use COVID-19, SARSCov-2, coronavirus, and metformin as search terms. We will search literatures in a combination of "Title/Abstract" and Medical Subject Headings (MeSH).

Participant or population: Patients with COVID-19 receiving conventional treatments with and those without metformin medication.

Intervention: Patients with COVID-19 receiving metformin medication in combination with conventional treatments.

Comparator: Patients with COVID-19 receiving conventional treatments alone.

Study designs to be included: We will analyze perspective studies including cohort studies, randomized controlled trials (RCTs), and non-RCTs, together along with retrospective studies, reported in both English and Chinese. Reviews, editorial materials and meeting abstracts will be excluded.

Eligibility criteria: Perspective or retrospective studies that analyzed the outcomes of patients with COVID-19 treated with and without metformin.

Information sources: PubMed, Embase, Cochrane Library, China National Knowledge Infrastructure (CNKI), Wan fang, and Chinese Biomedical Literature Database (CBM), from its establishment to Dec 31, 2020.

Main outcome(s): The outcomes include death, intubation, admission to ICU, deterioration, and the length of stay. Safety indicators include gastrointestinal tract adverse events, lactic acidosis and any adverse events.

Data management: Once the study is eligible, we will collect the following data: basic information (name of the first author, region of study, year of publication, study design), participants (numbers, race, age, gender, follow-up duration), and outcomes (death, intubation, admission to ICU, deterioration, length of stay, adverse events). We will contact the corresponding authors to deal with the missing or unclear data and then remove the study if any data is not available.

Quality assessment / Risk of bias analysis: We will use Cochrane Collaboration's bias risk tool containing 7 items divided into 3 categories (high risk, low risk, uncertain risk) to evaluate the risk of bias for RCTs and non-RCTs. For other type of study, we will use Newcastle-Ottawa Scale (NOS) and the results are divided into 3 grades according to the scores (good: 7-9, fair: 5-6, poor: 0-4).

Strategy of data synthesis: We will analyze the data using Review Manager 5.3 (Nordic Cochran Centre, Copenhagen, Denmark). We will calculate the odds ratio, relative risk, or hazard ratio, and its $95 \%$ confidence interval (CI) for binary data such as gender, death, intubation, admission to ICU, deterioration, and adverse events. We will calculate the mean difference for continuous data such as age, and length of stay. We will access the heterogeneity using Cochrane Q-test and 12 statistics. We will use a fixed effect 
model if the value of 12 under $50 \%$ or $\mathbf{P}$ beyond 0.1 and a random-effect model if the value of $\mathrm{I} 2$ beyond $50 \%$ or $\mathrm{P}$ under 0.1 .

Subgroup analysis: We will perform a subgroup analysis based on the following aspects: 1) Age: study with average baseline age over 65 yrs versus no larger than 65yrs; 2) Diabetes: study including individuals with and without underlying diabetes; 3) Obesity: study including individuals with (BMI $<28 \mathrm{~kg} / \mathrm{m} 2)$ and without obesity (BMI $\geq 28 \mathrm{~kg} / \mathrm{m} 2$ ).

Sensitivity analysis: We will perform the sensitivity analysis by removing 1 study each time from pooled analysis and reporting the results in a summary table.

Language: English or Chinese.

Country(ies) involved: China.

Keywords: COVID-19, SARS-CoV-2, metformin, clinical outcomes, adverse effects, efficacy.

Contributions of each author:

Author 1 - Yin Li.

Author 2 - Tong Sun.

Author 3 - Xue Yang.

Author 4 - Peijing Yan.

Author 5 - Zhi Zeng. 